\title{
Design of Feed-forward Controller with Stick-slip Friction Modeling in Electro-mechanical Brake System
}

\author{
Giseo Park, Sanghun Jung and Seibum Choi \\ Department of Mechanical Engineering, Korea Advanced Institute of Science and Technology, Daejeon, Korea
}

\begin{abstract}
Electro-Mechanical Brake $(E M B)$ is expected to be one of the future brake system. Feedback controller with sensor measuring is commonly used for control of EMB. However, this controller has some issues like delayed response and extra cost about sensor installation. In this paper, Feed-forward controller in EMB is proposed for solving these problems of feedback control. Also, it is very necessary to describe dynamical phenomenon of friction in actual EMB system. The actual EMB system shows stick-slip friction of mechanical parts which is difficult to model and apply to design of controller. This research is focused on exquisitely describing this stick-slip friction. In order to do this, the experiment about EMB is proceeded in the open loop system with the motor current command and data from the experiment is used for identification of model parameters during stiction. Then, parameters during slip is estimated in the closed loop system. Finally, developed friction model of EMB is proposed and it is utilized for design of feed-forward controller. Matlab Simulink is used for design of EMB simulation model and EMB test bench is utilized for experiment. Performance of proposed control system is compared with that of feedback control system.
\end{abstract}

\section{Introduction}

Brake-By-Wire (BBW) system is expected to be one of the future brake system [4]. Because it has many advantages like faster response, shorter braking distance, good space efficiency and connectivity to vehicle chassis control systems like Anti-lock Brake System (ABS) and Electronic Stability Control (ESC) [7]. This system has no direct connection between brake pedal and wheel. Then, hydraulic line in hydraulic brake system is replaced by pedal sensor, brake pedal simulator, control unit and back-up system. Electro-Mechanical Brake (EMB) is one of the BBW system and has been studied in many companies in automotive industry [5].

Most of EMB is controlled by feedback controller embedded in ECU [3]. The control system has cascaded architectures, which are consisted of force controller, velocity controller, and current controller in series [1]. The cascaded architecture is shown in Fig. 1. However, this controller has some issues related to control performance and usefulness. Because controller based feedback, which is measured value by a sensor, cannot help having delayed response. This delayed response has a bad effect for control performance.

In this paper, new EMB controller design using feedforward control is proposed for solving these issues of existing feedback controller. It is anticipated to have no delayed response.

Also, it is very necessary to utilize clamping force and friction torque estimator for generating feed-forward term.
EMB simulation model is developed and used for accuracy and usefulness of this control system are shown with comparison of the simulation results in feedback control system.

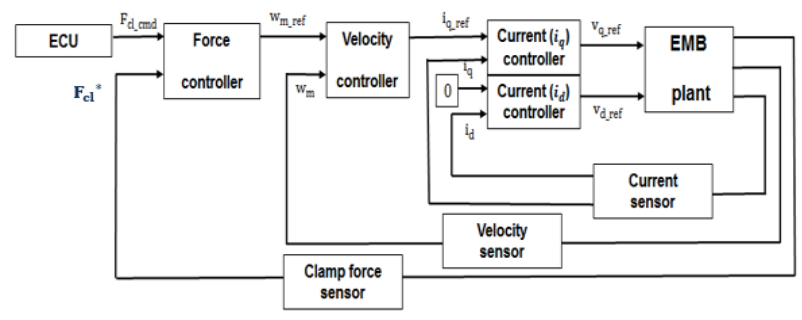

Figure 1. Cascaded architecture of EMB feedback controller

\section{EMB dynamics}

\subsection{Simplified EMB model}

The simplified model of EMB plant is necessary for controller design based on characteristics of EMB plant. Fig. 2 shows diagram of this model. Torque balance equation of EMB model is described as follows.

$$
\mathrm{T}_{\mathrm{m}}=\mathrm{T}_{\mathrm{L}}+\mathrm{J}_{\mathrm{tot}} \frac{\mathrm{d}^{2}}{\mathrm{dt}^{2}} \theta_{\mathrm{m}}+\mathrm{T}_{\mathrm{f}}
$$

$\mathrm{T}_{\mathrm{m}}$ means motor torque, which is generated by Permanent Magnet Synchronous Motor (PMSM) or Brushless DC motor. These motors are commonly used 
for motor of EMB. Also It can be expressed like that motor current is multiplied by motor-torque constant $\mathrm{K}_{\mathrm{m}}$ [6].

$$
\mathrm{T}_{\mathrm{m}}=\mathrm{K}_{\mathrm{m}} \mathrm{i}_{\mathrm{q}}
$$

$\mathrm{T}_{\mathrm{L}}$ is load torque, which makes clamping force on brake disk. Rotational motion of motor is converted into linear motion by the ball screw. $p$ is pitch of ball screw and $\eta$ is efficiency of ball screw. Also, GR is total gear ratio, which can be calculated from the number of gear teeth of all reduction gears in EMB. Load torque is represented as follows [1]:

$$
\mathrm{T}_{\mathrm{L}}=\frac{1}{\mathrm{GR}} \frac{\mathrm{p}}{2 \pi \eta} \mathrm{F}_{\mathrm{cl}}=\mathrm{k}_{\mathrm{cl}} \mathrm{F}_{\mathrm{cl}}
$$

$\mathrm{T}_{\mathrm{f}}$, friction torque will be explained in chapter 3 . Friction torque modeling.

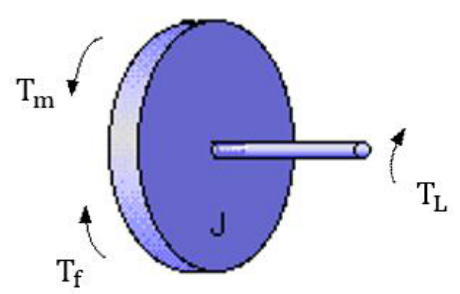

Figure 2. Diagram of Simplified EMB model

\subsection{Clamping force estimation}

It is recommended that a clamping force sensor is replaced with simple and precise algorithm due to high price of the sensor. Also, the sensor has low accuracy in high temperature and structural issue. Clamping force algorithm has been studied in a lot of researches $[1,2,4]$. In previous papers, clamping force is expressed as a function of motor angle or displacement of brake pad [2].

In this paper, clamping force versus motor angle is estimated with only measuring motor current and motor angle. In order to estimate clamping force, motor current versus motor angle is expressed by fitted curve, which is second-order polynomial function.

The curve of motor current is distinguished between clamping and releasing situations. In each situation, rotation of motor angle has forward and backward directions. If same motor angle in these two situations, each friction torque has same absolute value and different sign. Because friction toque in EMB has features of Coulomb friction and contain no viscous friction. In spite of the opposite direction, if the motor angle is same, the clamping force is equal. Clamping force in each situation is represented by the following equations.

$$
\begin{aligned}
& \widehat{\mathrm{F}}_{\mathrm{cl}}\left(\theta_{\mathrm{m}}\right)=\frac{\mathrm{K}_{\mathrm{m}} \mathrm{i}_{\mathrm{q}-\mathrm{cl}}-\mathrm{J}_{\mathrm{tot}} \frac{\mathrm{d}^{2}}{\mathrm{dt}^{2}} \theta_{\mathrm{m}-\mathrm{cl}}-\mathrm{T}_{\mathrm{f}}}{\mathrm{k}_{\mathrm{cl}}} \\
& \widehat{\mathrm{F}}_{\mathrm{cl}}\left(\theta_{\mathrm{m}}\right)=\frac{\mathrm{K}_{\mathrm{m}} \mathrm{i}_{\mathrm{q}-\mathrm{rel}}-\mathrm{J}_{\mathrm{tot}} \frac{\mathrm{d}^{2}}{\mathrm{dt}^{2}} \theta_{\mathrm{m}-\mathrm{rel}}+\mathrm{T}_{\mathrm{f}}}{\mathrm{k}_{\mathrm{cl}}}
\end{aligned}
$$

Calculating average between (4) and (5), clamping force of any motor angle $\theta_{\mathrm{m}}$ can be derived. Also, there is no consideration of friction torque $T_{f}$, which is estimated in chapter 3 Friction torque modeling.

$$
\widehat{\mathrm{F}}_{\mathrm{cl}}\left(\theta_{\mathrm{m}}\right)=\frac{\mathrm{K}_{\mathrm{m}}\left(\mathrm{i}_{\mathrm{q}-\mathrm{cl}}+\mathrm{i}_{\mathrm{q}-\mathrm{rel}}\right)-\mathrm{J}_{\mathrm{tot}}\left(\frac{\mathrm{d}^{2}}{\mathrm{dt}^{2}} \theta_{\mathrm{m}-\mathrm{cl}}+\frac{\mathrm{d}^{2}}{\mathrm{dt}^{2}} \theta_{\mathrm{m}-\mathrm{rel}}\right)}{2 \mathrm{k}_{\mathrm{cl}}}
$$

Fig. 3 shows this process and estimated clamping force $\hat{F}$ versus motor angle. Characteristic curve is estimated with kissing point, which means zero value of the curve and calculated by measurement when pad contacts brake disk. In order to verificate this algorithm, experiment data is necessary.

Thus, EMB test bench is designed and composed of EMB hardwrare, ECU, Micro autobox and control desk in Fig. 4.

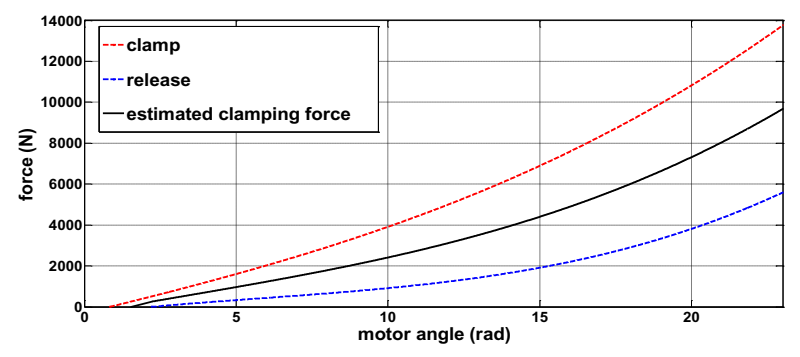

Figure 3. EMB characteristic curve

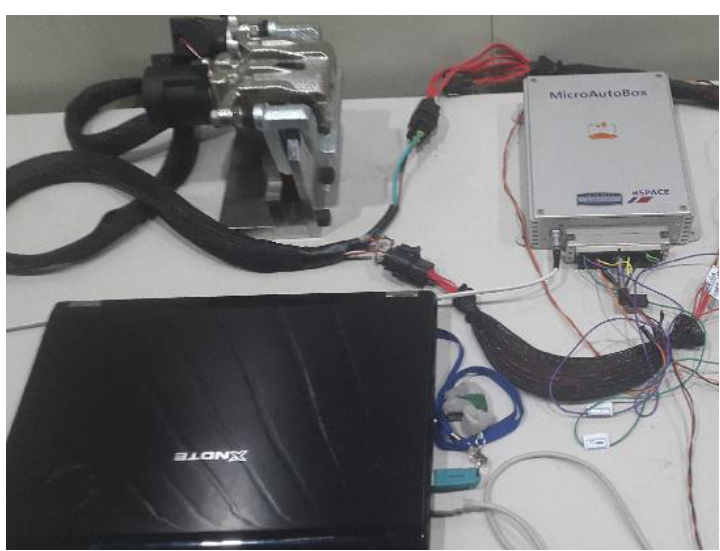

Figure 4. EMB test bench

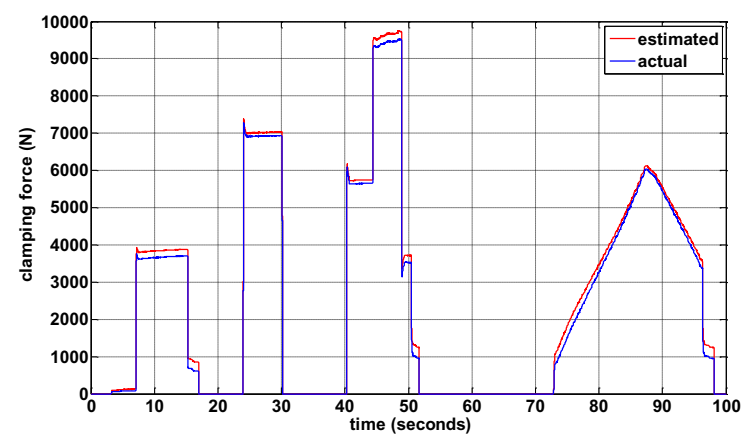

Figure 5. Estimated clamping force results

Fig. 5 represents real time esimation results of clamping force. In Fig. 5, compared to actual clamping force measured by sensor, estimated clamping force has contentable root mean square error, $0.3 \mathrm{kN}$. clamping force esimation algorithm is utilized for design of feedforward controller. 


\section{Friction torque modeling}

Friction toque occuring in motor, gear and ball screw of EMB is complicated to define as mathmatical expression. However, estimation of friction torque is necessary for elaborate design of EMB feed-forward control system. Chris Line proposed friction toque model of EMB [3]. According to motor angular velocity, friction torque is divided to static and kinetic friction torque. Also, the friction torque is described as a function of clamping force and angular velocity.

In this paper, modificated friction torque model is proposed for exquisitely describing real dynamics of EMB. Especially, accuracy of modeling of kinetic friction torque is improved through clamping force estimation. Following expression shows this modeling.

$$
\mathrm{T}_{\mathrm{f}, \mathrm{k}}\left(\theta_{\mathrm{m}}\right)=\left\{\begin{array}{l}
\operatorname{sign}\left(\mathrm{w}_{\mathrm{m}}\right)\left\{\mathrm{T}_{\mathrm{f} 0}+\alpha\left(\theta_{\mathrm{m}}-\hat{\theta}_{0}\right)^{2}+\beta\left(\theta_{\mathrm{m}}-\hat{\theta}_{0}\right)\right\} \\
\mathrm{T}_{\mathrm{f} 0} \operatorname{sign}\left(\mathrm{w}_{\mathrm{m}}\right)
\end{array}\right.
$$

Upper expression in (7) is occured when motor angle is over kissing point. Lower expression in (7) is occured when motor angle is under kissing point. Because friction torque is proportional to clamping force, friction torque can be expressed by second order polynomial function of motor angle. Also, before generating clamping force such as under kissing point, friction torque is almost constant.

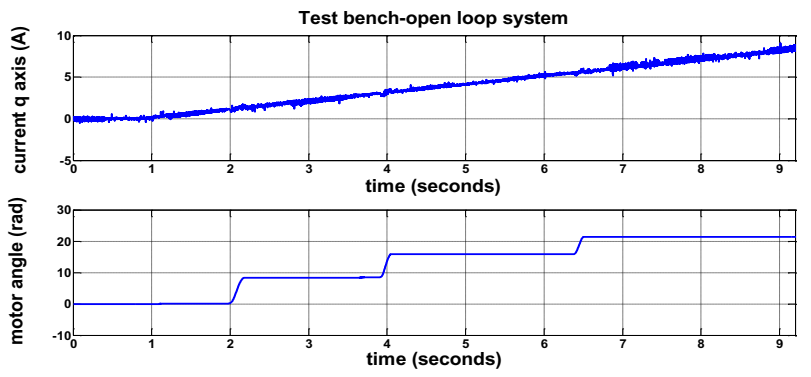

Figure 6. Stick-slip phenomenon of actual EMB
Fig. 6 shows stick slip phenomenon, which means spotaneous jerking motion. The rotation part of EMB slip over the surface when external torque is larger than friction torque. This external torque is motor torque minus load torque and increased can be increased by motor current command such as Fig. 6. The rotation stops when the external torque is smaller than friction torque. Because friction torque is nonlinearly increased according to motor angle, more larger torque is determined alternately between external torque and friction torque. Therefore, stick and slip motion of rotation part is occured alternately and repeatedly.

If a designed simulation model contains frcition torque model, which can express stick slip phenomenon, the simulation model can be identified as very exquisite model similar to actual EMB plant. In order to make this model, unknown parameters in (7), $\mathrm{T}_{\mathrm{f} 0}, \alpha, \beta$ are estimated using actual experiment data like Fig. 6. Least square method is utilized for estimation of these parameters. Fig. 7 shows block diagram of friction torque in EMB simulation model.

\section{Design of feed-forward controller}

Using clamping force estimator and identified friction torque model, EMB feed-forward controller is designed. Clamping force estimator is transformed to inverse function, which yields the motor angle value according to desired clamping force. Feed-forward term equal additional motor current command. It is designed like follow equation.

$$
\mathrm{i}_{\mathrm{q}-\mathrm{ff}}=\frac{\mathrm{J} \dot{\mathrm{w}}+\gamma \mathrm{F}_{\mathrm{cl}}+\mathrm{T}_{\mathrm{f}}}{\mathrm{k}_{\mathrm{t}}}
$$

Compared with feedback control system in Fig. 1, feed-forward term is added and clamping force sensor is replaced to characteristic curve. Diagram of feed-forward control system is shown in Fig. 8.

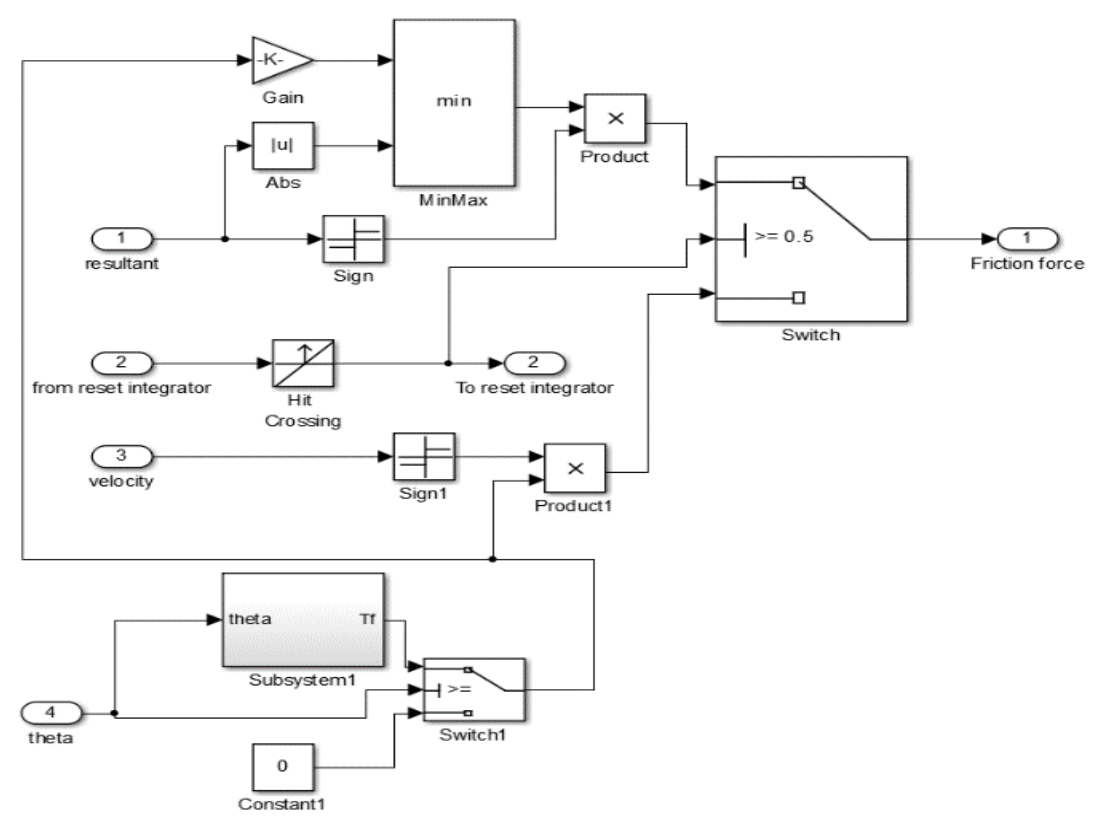

Figure 7. Simulation model of EMB friction torque 


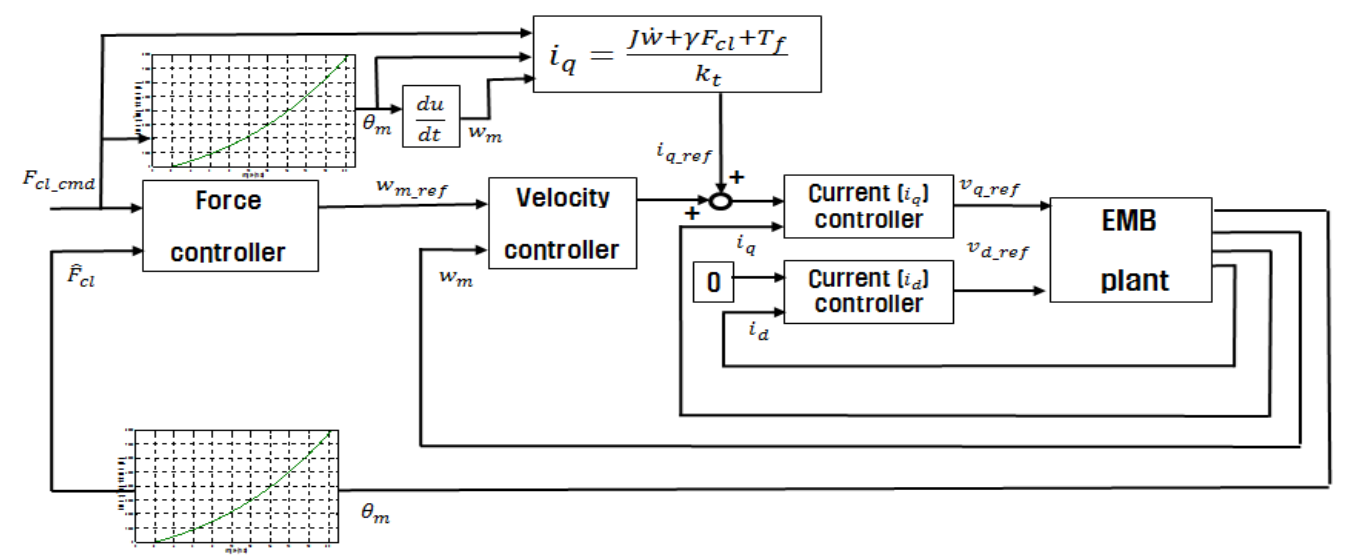

Figure 8. Diagram of feed-forward control system

\section{Simulation results}

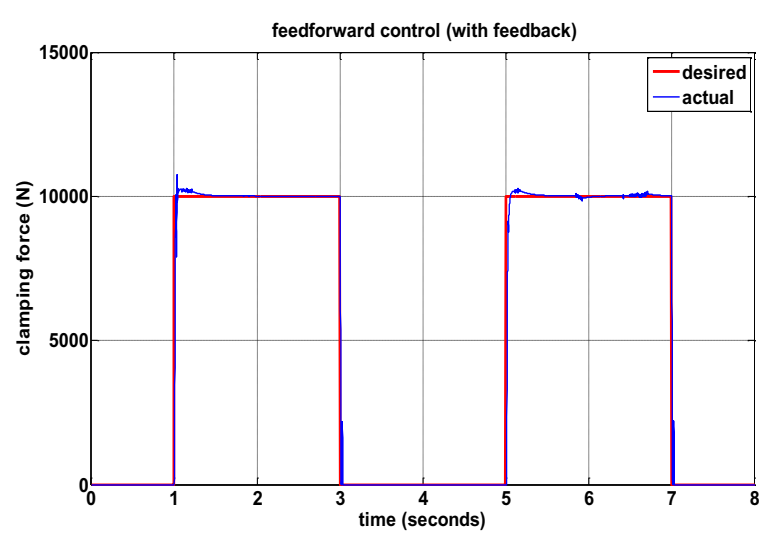

Figure 9. Diagram of feed-forward control system

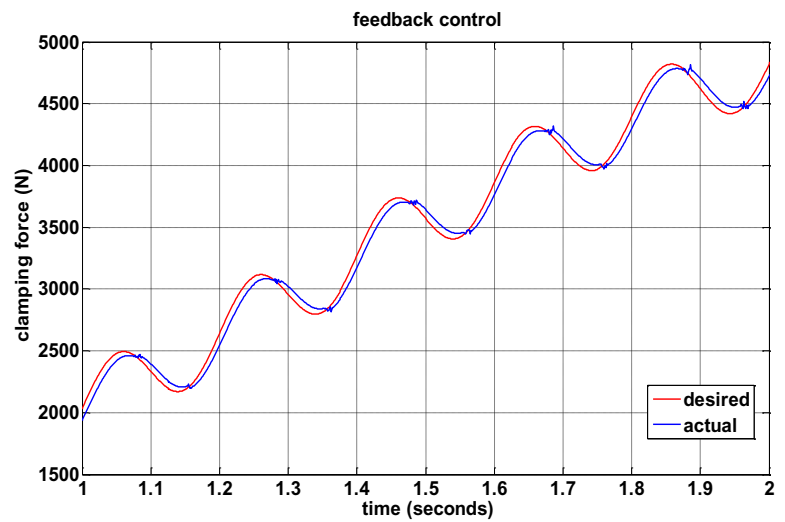

Figure 10. Control results in feedback control system

The control system is implemented in Matlab Simulink and performance of controller is evaluated. In Fig. 9, maximum step input is commanded and it is shown that actual clamping force are close to desired clamping force. As shown in Fig. 10 and Fig. 11, performance of feedforward control system is better than that of feedback control system in terms of same sinusoidal input. Rms errors in each systems are $0.15 \mathrm{kN}$ and $0.5 \mathrm{kN}$.

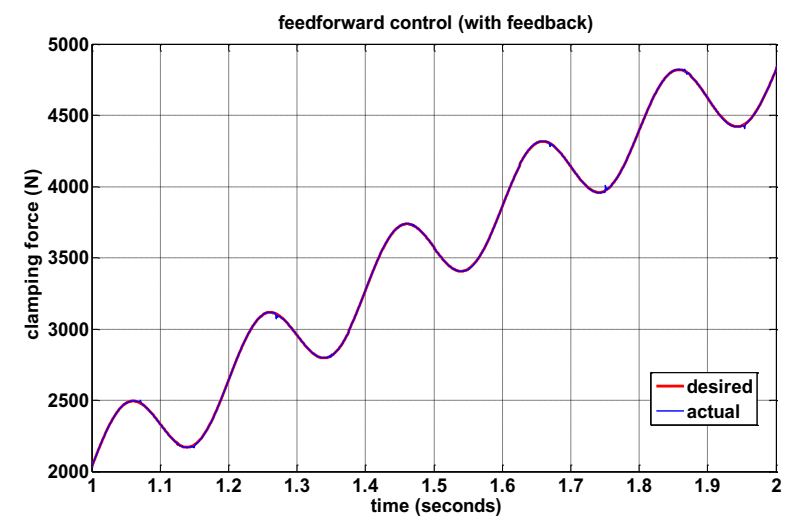

Figure 11. Control results in feed forward control system

\section{Conclusion}

In this paper, EMB feed-forward control system using clamping force estimator and friction torque model is proposed. Especially, friction torque model exquisitely describes actual EMB plant using experimental data in stick slip phenomenon. Feed-forward controller is effective to solve some problems of existing EMB feedback control system like control performance. Feedforward term related to clamping force, inertia torque and friction torque is very effective to follow desired input with no delay. It is anticipated that these algorithm can be used for improvement of EMB system.

\section{References}

1. R. Schwarz, R. Isermann, J. Bohm, J. Nell, P. Rieth, SAE 980600 (1998)

2. Y. H. Ki, K. J. Lee, J. S. Cheon, H. S. Ahn, Int. J. Automotive. Tech. 14, 739 (2013)

3. C. Line, C. Manzie, M. C. Good, IEEE Trans. Control Sys. Tech. 16, 446 (2008)

4. C. Jo, S. Hwang, H. Kim, IEEE Trans. Vehicular Tech. 59, 3205 (2010)

5. J. Choi, I. Kim, K. Huh, M. Kim, J. Kim. K. Kim, KSAE 653 (2009) 
6. P. Pillay, R. Krishnan, IEEE Trans. Industry Applications, 25, 265 (1989)

7. S. Saric, A. Bab-Hadiashar, R. Hoseinnezhad, IEEE Trans. Vehicular Tech. 57, 778 (2008)
8. R. Hoseinnezhad, A. Bab-Hadiashar, T. Rocco, IEEE Trans. Vehicular Tech. 57, 770 (2008)

9. R. Hoseinnezhad, A. Bab-Hadiashar, IEEE Trans. Industry Elec. 54, 1052(2007) 